\title{
Formal Foundations for Aspects
}

\author{
Radha Jagadeesan \\ DePaul University, USA \\ rjagadeesan@cs.depaul.edu
}

\begin{abstract}
Aspects have emerged as a powerful tool in the design and development of systems. Aspect-orientation ideas are paradigm independent and have been developed for object-oriented, imperative and functional languages.

This talk will discuss a suite of results that aim to level the foundational playing field between aspects and other programming paradigms. In this context, we will argue that aspects are no more intractable than stateful higher order programs.

The talk is based on joint work with Glenn Bruns, Alan Jeffrey, Corin Pitcher and James Riely.
\end{abstract}

